\title{
TIDES IN ROTATING FLUIDS
}

\author{
PeTer Goldreich \\ California Institute of Technology \\ AND \\ Philip D. NichOlson \\ Cornell University \\ Received 1988 September 8; accepted 1988 December 29
}

\begin{abstract}
We consider the tidal disturbance forced in a differentially rotating fluid by a rigidly rotating external potential. The fluid is assumed to be inviscid, insulated, and self-gravitating, and to have laminar unperturbed and perturbed velocity fields. The external potential may exert a steady torque on the fluid which is of second order in its strength. However, to this order, we prove that there are no secular changes in the angular momenta of fluid particles, except possibly at corotation where the angular velocity, $\Omega(r, \theta)$, is equal to the pattern speed of the potential, $\Omega_{p}$. A corollary of our theorem is that, except at corotation, all of the angular momentum transferred to the fluid by the external potential must be transported away by internal stresses. In the applications of which we are aware, these stresses are associated with waves.
\end{abstract}

Subject headings: hydrodynamics - rotation

\section{INTRODUCTION}

Stars, planetary rings, and disk galaxies are rotating systems that exhibit fluid behavior in appropriate contexts and may suffer tidal forcing.

Zahn (1975, 1977) demonstrated that tidal forcing of an early-type star excites gravity waves at the boundary between its convective core and radiative envelope. The gravity waves propagate outward to the stellar surface where, as a consequence of radiative damping, they are only partially reflected. Zahn estimated the tidal torque associated with the radiatively damped gravity waves and argued that it is compatible with that deduced from observations of tidal evolution in close binary stars.

Goldreich and Tremaine $(1978,1979)$ showed that satellites excite spiral density waves in thin disks. The wave trains begin at Lindblad resonances and propagate toward corotation. Subsequently, many spiral wave trains were discovered in Saturn's rings by the Voyager spacecraft.

The examples of tidally forced wave motion cited above have a number of common features. In both, the external potential varies slowly on the scale of the wavelengths of the waves which it excites. For this reason, the potential couples effectively to the waves only at turning points where the WKBJ wavelength is formally infinite. Turning points separate regions within which the waves propagate from those within which they are evanescent. The net tidal torque is exerted where the wave train is excited. The theorem we prove below establishes that internal stresses transport away all of the angular momentum which the tidal torque transfers to the fluid. In the examples referred to above, the internal stresses are associated with waves. The angular momentum is ultimately deposited where the waves damp.

Consider our first example, the tidal forcing of an early-type star. Gravity waves can propagate in the stably stratified radiative envelope but not in the unstably stratified convective core. Thus, the outer boundary of the core is a turning point for gravity waves, and that is where the net tidal torque is exerted.
Our second example involves tidal forcing of spiral density waves in thin disks by satellites. These density waves are supported by self-gravity and their propagation is restricted to the region between Lindblad resonance and corotation. Thus, the Lindblad resonance is a turning point for the waves, and that is where the net satellite torque is applied.

In specific cases it is possible to solve the tidal perturbation equations and then to calculate the transport of angular momentum by waves. Such a program has been carried out for tidally excited density waves in thin disks by Goldreich and Tremaine $(1978,1979)$. They proved that density waves transport away all of the angular momentum supplied by the tidal torque. A similar result seems likely to apply to the tidal forcing of early-type stars, but a proof is lacking. Our investigation of this problem led us to the proof of the theorem given in $\S \mathrm{II}$, which is the central result of this paper. The theorem is quite general and demonstrates the underlying structure common to many examples of tidal forcing in conservative systems. A brief discussion of an analogous theorem in celestial mechanics and of our theorem's physical interpretation is provided in $\S$ III. The theorem is applied to resolve a puzzling set of observations related to tidal evolution in early-type stars in a companion paper (Goldreich and Nicholson 1989)

\section{THE THEOREM}

We consider tidal perturbations of a stationary, axially symmetric, differentially rotating, self-gravitating, fluid body. The fluid is assumed to be both inviscid and insulated and to obey a $\gamma$-law equation of state. Moreover, throughout our analysis we assume that both the unperturbed and perturbed velocity fields are laminar.

We adopt a spherical coordinate system $(r, \theta, \phi)$. The dependent variables that characterize the fluid are its velocity, $v$, density, $\rho$, pressure, $p$, and self-gravitational potential, $\psi$. In the unperturbed state, $\boldsymbol{v}=v_{\phi}(r, \theta) \hat{\boldsymbol{e}}_{\phi}$. form

The external (sourceless) potential is assumed to take the

$$
U(r, \theta, \phi ; t)=U_{l} r^{l} P_{m}^{l}(\cos \theta) \cos m\left(\phi-\Omega_{p} t\right),
$$


where $P_{l}^{m}$ is an associated Legendre function. The constant magnitude $U_{l}$ sets the scale for the strength of the perturbations. We assume that $\epsilon \equiv O\left(U_{l} / \psi\right) \ll 1$.

The external potential rotates rigidly with pattern speed $\Omega_{p}$; its inertial oscillation frequency is $\omega=m \Omega_{p}$. In the frame of the fluid the frequency is Doppler-shifted to

$$
\sigma(r, \theta) \equiv m\left(\Omega_{p}-\Omega\right),
$$

where

$$
\Omega(r, \theta)=\frac{v_{\phi}(r, \theta)}{r \sin \theta} .
$$

Corotation resonances are located where $\sigma(r, \theta)=0$. They play a special role in the dynamics.

\section{a) Lagrangian Decomposition}

The hydrodynamic equations of motion follow from the application of Hamilton's principle to the Lagrangian density

$$
\mathscr{L}=\rho \frac{|\boldsymbol{v}|^{2}}{2}-\frac{p}{(\gamma-1)}-\rho \psi-\rho U-\frac{|\nabla \psi|^{2}}{8 \pi G} .
$$

Eularian and Lagrangian implementations of the variation principle are described by Newcomb (1962). In both cases, the fundamental variation is to the position, $\boldsymbol{x}$, of each fluid element. The constraints implied by the equation of continuity and the equation of state are included by relating the variations of $\rho$ and $p$ to those of $\boldsymbol{x}$. These relations are local ones. The situation for $\psi$ is a bit more complicated; its variation is related that of $\rho$, and hence $\boldsymbol{x}$, in a nonlocal manner through the solution of Poisson's equation. Accordingly, we vary $\psi$ independently of $\boldsymbol{x}$, which yields Poisson's equation.

Next, we reduce $\mathscr{L}$ to a form suitable for describing linear, tidally induced, perturbations. To do so, we follow a procedure outlined by Dewar (1970) in his extension of earlier work by Whitham (1965). The exact state, $\left(\rho^{*}, p^{*}, v^{*}, \psi^{*}\right)$, is decomposed into a background state, $(\rho, p, v, \psi)$, and a perturbation obtained by displacing the position of each fluid element from $\boldsymbol{x}$ to $\boldsymbol{x}^{*}=\boldsymbol{x}+\boldsymbol{\xi}$. The critical insight provided by Dewar (1970) is to define $\xi$ such that its time average following the background motion of a fluid particle, $\langle\xi\rangle$, vanishes. The implications of this choice will become apparent shortly. We differ from Dewar (1970) in that we do not suppose that the perturbation describes a purely wave-like disturbance.

The background state is uniquely defined by the requirement that $\langle\xi\rangle$ vanish; it is not to be confused with the unperturbed state. In $\S$ II $b$, we show that the background state may exhibit both periodic and secular variations with leading terms of each type being of order $\epsilon^{2}$.

The displacement field defines a transformation from the background state to the exact state whose Jacobian is given by

$$
J(x, t)=\operatorname{Det}\left(1+\frac{\partial \xi}{\partial x}\right) .
$$

To second order in $\xi$

$$
J \approx 1+\nabla \cdot \xi-\frac{1}{2}\left[\nabla \xi: \nabla \xi-(\nabla \cdot \xi)^{2}\right] .
$$

The double dot product $\boldsymbol{a b}: \boldsymbol{c d} \equiv(\boldsymbol{b} \cdot \boldsymbol{c})(\boldsymbol{a} \cdot \boldsymbol{d})$. The changes in $v, \rho$, and $p$ associated with this transformation read

$$
\begin{gathered}
v^{*}=v+\frac{d \xi}{d t}, \\
\rho^{*}=\frac{\rho}{J},
\end{gathered}
$$

and

$$
p^{*}=\frac{p}{J^{\gamma}} .
$$

The time derivative which appears in equation (7) accounts for the total variation of $\xi$ following the background motion of a fluid particle. Thus,

$$
\frac{d \xi}{d t}=\frac{\partial \xi}{\partial t}+v \cdot \nabla \xi
$$

The transformation of $\psi$ is more subtle since it has a nonlocal dependence on $\xi$. We define by $\Delta \psi$ the Langrangian change of the gravitational potential felt by a fluid particle,

$$
\Delta \psi(\boldsymbol{x}, t) \equiv \psi^{*}(\boldsymbol{x}+\boldsymbol{\xi}, t)-\psi(\boldsymbol{x}, t) .
$$

It is related to the Eulerian potential variation,

$$
\delta \psi(\boldsymbol{x}+\boldsymbol{\xi}, t) \equiv \psi^{*}(\boldsymbol{x}+\boldsymbol{\xi}, t)-\psi(\boldsymbol{x}+\boldsymbol{\xi}, t),
$$

by

$$
\Delta \psi(x, t)=\delta \psi(x+\xi, t)+\psi(x+\xi, t)-\psi(x, t) .
$$

To second order in $\xi$

$$
\psi(x+\xi, t)-\psi(x, t) \approx \xi \cdot \nabla \psi+\frac{1}{2} \xi \xi: \nabla \nabla \psi
$$

We now have assembled all of the ingredients needed for the perturbation expansion of $\mathscr{L}$. We write

$$
L=\int d^{3} x^{*} \mathscr{L}^{*}=\int d^{3} x\left[\mathscr{L}_{0}+\mathscr{L}_{1}+\mathscr{L}_{2}\right],
$$

where the volume element $d^{3} x^{*}=J d^{3} x$. The gravitational selfenergy term is most conveniently transformed by a change of the dummy variable of integration from $x^{*}$ to $x$. The other terms in $\mathscr{L}$ are reduced using the relations given by equations (5)-(14). A straightforward calculation yields

$$
\begin{gathered}
\mathscr{L}_{0}=\rho \frac{|v|^{2}}{2}-\frac{p}{(\gamma-1)}-\rho \psi-\frac{|\nabla \psi|^{2}}{8 \pi G} \\
\mathscr{L}_{1}=\rho v \cdot \frac{d \xi}{d t}+p \nabla \cdot \xi-\rho \xi \cdot \nabla \psi-\rho \delta \psi-\rho U-\frac{\nabla \psi \cdot \nabla \delta \psi}{4 \pi G} \\
\mathscr{L}_{2}=\frac{\rho}{2}\left|\frac{d \xi}{d t}\right|^{2}-\frac{p}{2}\left[(\gamma-1)(\nabla \cdot \xi)^{2}+\nabla \xi: \nabla \xi\right]-\rho \xi \cdot \nabla \delta \psi \\
-\frac{\rho \xi \xi}{2}: \nabla \nabla \psi-\rho \xi \cdot \nabla U-\frac{|\nabla \delta \psi|^{2}}{8 \pi G}
\end{gathered}
$$

The Euler-Lagrange equations,

$$
\frac{\partial}{\partial t} \frac{\partial \mathscr{L}}{\partial(\partial \xi / \partial t)}+\nabla \cdot \frac{\partial \mathscr{L}}{\partial \nabla \xi}-\frac{\partial \mathscr{L}}{\partial \xi}=0
$$

applied to $\mathscr{L}_{1}$ and $\mathscr{L}_{2}$ yield, respectively, the unperturbed equation of motion and the linearized perturbation equations. Poisson equations for $\psi$ and $\delta \psi$ are recovered from the EulerLagrange equation

$$
\nabla \cdot \frac{\partial \mathscr{L}}{\partial \nabla \delta \psi}-\frac{\partial \mathscr{L}}{\partial \delta \psi}=0,
$$

applied to $\mathscr{L}_{1}$ and $\mathscr{L}_{2}$, respectively. The former is an identity, and the latter, which relates $\delta \psi$ to $\xi$,

$$
\nabla^{2} \delta \psi=4 \pi G \nabla \cdot \rho \xi,
$$

shows that $\langle\delta \psi\rangle=0$ as a consequence of $\langle\xi\rangle=0$. 


\section{b) Background Evolution}

To derive the equation of motion for the background, we vary $\boldsymbol{x}$, the background position of the particles, in $\mathscr{L}$. The associated Eulerian variations of $v, p$, and $\rho$ are given by

$$
\begin{gathered}
\delta v=\frac{d}{d t} \Delta x-\Delta x \cdot \nabla v, \\
\delta \rho=-\rho \nabla \cdot \Delta x-\Delta x \cdot \nabla \rho, \\
\delta p=-\gamma p \nabla \cdot \Delta x-\Delta x \cdot \nabla p .
\end{gathered}
$$

The tidal perturbations described by $\xi$ and $\delta \psi$ are treated as prescribed functions of $\boldsymbol{x}$ and $t$. The resulting Euler-Lagrange equations for the background read

$$
\begin{aligned}
\frac{\partial}{\partial t}\left(\frac{\partial \mathscr{L}}{\partial v}\right)+\nabla \cdot(v & \left.\frac{\partial \mathscr{L}}{\partial \boldsymbol{v}}-\rho \frac{\partial \mathscr{L}}{\partial \rho} \boldsymbol{I}-\gamma p \frac{\partial \mathscr{L}}{\partial p} \boldsymbol{I}\right) \\
& +\nabla \boldsymbol{v} \cdot \frac{\partial \mathscr{L}}{\partial \boldsymbol{v}}+\nabla \rho \frac{\partial \mathscr{L}}{\partial \rho}+\nabla p \frac{\partial \mathscr{L}}{\partial p}=0
\end{aligned}
$$

and

$$
\nabla \nabla: \frac{\partial \mathscr{L}}{\partial \nabla \nabla \psi}-\nabla \cdot \frac{\partial \mathscr{L}}{\partial \nabla \psi}+\frac{\partial \mathscr{L}}{\partial \psi}=0
$$

Here, $\mathscr{L}$ is treated as an explicit function of the variables $v, \rho, p$, $\psi, \nabla \psi$, and $\nabla \nabla \psi$ in forming the partial derivatives which appear in equations (25) and (26), and $I$ is the unit diadic. The operators $\partial / \partial t$ and $\nabla$ have their standard meanings, differentiation with respect to $t$ at fixed $\boldsymbol{x}$, and differentiation with respect to $\boldsymbol{x}$ at fixed $t$.

Our result is essentially that of Dewar (1970), with the addition of equation (26) which determines the background gravitational potential. With $\mathscr{L}_{0}$ substituted for $\mathscr{L}$, equations (25) and (26) yield, respectively, the unperturbed versions of the equations of motion and Poisson's equation. The reaction of the background to the tidal perturbations follows from adding the contributions obtained by inserting $\mathscr{L}_{1}$ and $\mathscr{L}_{2}$ into the same equations. The first-order terms vanish, as one might expect from the manner in which we decompose the exact state of the system into a background state plus a periodic perturbation. Verifying this assertion requires a straightforward but tedious calculation, which we leave to the reader. The main steps consist of reducing the first-order terms to zero through application of the linear perturbation equations which result from substituting $\mathscr{L}_{2}$ into the Euler-Lagrange equations (19) and (20).

To determine the background evolution to second order in the strength of the tidal perturbation would be a formidable undertaking. To begin, it would be necessary to solve the perturbation equations (19) and (20) to find $\xi(x, t)$ and $\delta \psi(x, t)$. These functions would then be substituted into equation (18) for $\mathscr{L}_{2}$, and the resulting expression used in equations (25) and (26). Finally, there would be the task of solving the partial differential equation of motion in conjunction with Poisson's equation. Fortunately, we have a less ambitious goal. What we are after is the steady, or secular, evolution of the background fluid; short time scale variations are of no interest to us.

To proceed, we write the perturbation and background fields in the form of Fourier series in the variable $\phi-\Omega_{p} t$. For the perturbations, the leading terms read

$$
\xi=\tilde{\xi} \cos m\left(\phi-\Omega_{p} t+\alpha\right)
$$

and

$$
\delta \psi=\delta \tilde{\psi} \cos m\left(\phi-\Omega_{p} t+\beta\right) .
$$

We have established that the leading background variations are of second order in $\epsilon$ so we may express an arbitrary background field, $Q$, as

$$
Q=\langle Q\rangle+\epsilon^{2} \tilde{Q} \cos 2 m\left(\phi-\Omega_{p} t+\eta\right) .
$$

Here, $\tilde{\xi}, \alpha, \delta \tilde{\psi}, \beta, \tilde{Q}, \eta$, and $\langle Q\rangle$ are functions of $\left(r, \theta ; \epsilon^{2} t\right){ }^{1}$

Next, we substitute the Fourier series expansions into equation (18) for $\mathscr{L}_{2}$ and insert the resulting expression into equations (25) and (26). Finally, we average over time following the unperturbed motion of the fluid particles. That is, we make the substitution $\phi-\Omega_{p} t=\phi_{0} \bar{\sigma} \sigma$ and perform a local time average holding $\tilde{\xi}, \alpha, \delta \tilde{\psi}, \beta, \tilde{Q}, \eta, \phi_{0}$, and $\langle Q\rangle$ constant. $^{2}$ This procedure yields the time-averaged equation of motion for the background

$$
\begin{aligned}
\langle\rho\rangle & \frac{d\langle\boldsymbol{v}\rangle}{d t}+\nabla\langle\rho\rangle+\langle\rho\rangle \nabla\langle\psi\rangle=-\langle\boldsymbol{v}\rangle \cdot \nabla \frac{\partial\left\langle\mathscr{L}_{2}\right\rangle}{\partial\langle\boldsymbol{v}\rangle} \\
& -\nabla\langle\boldsymbol{v}\rangle \cdot \frac{\partial\left\langle\mathscr{L}_{2}\right\rangle}{\partial\langle\boldsymbol{v}\rangle}+\langle\rho\rangle \nabla \frac{\partial\left\langle\mathscr{L}_{2}\right\rangle}{\partial\langle\rho\rangle}+\gamma\langle p\rangle \nabla \frac{\partial\left\langle\mathscr{L}_{2}\right\rangle}{\partial\langle p\rangle} \\
& +(\gamma-1) \nabla\langle p\rangle \frac{\partial\left\langle\mathscr{L}_{2}\right\rangle}{\partial\langle p\rangle},
\end{aligned}
$$

and Poisson's equation for the time averaged background potential

$$
\nabla^{2}\langle\psi\rangle=4 \pi G\langle\rho\rangle-4 \pi G \nabla \nabla: \frac{\partial\left\langle\mathscr{L}_{2}\right\rangle}{\partial \nabla \nabla\langle\psi\rangle} .
$$

The averaging procedure effects a great simplification of the background equations. We achieve an additional simplification by restricting our attention to the secular variation of the angular momentum in the background state. The scalar angular momentum of a particle, per unit mass, is given by

$$
h \equiv e_{z} \cdot(x \times v),
$$

where $e_{z}$ is the unit vector along the rotation axis. Differentiation with respect to time yields

$$
\frac{d h}{d t}=e_{z} \cdot\left(\frac{d x}{d t} \times v+x \times \frac{d v}{d t}\right)=r \sin \theta \hat{e}_{\phi} \cdot \frac{d v}{d t} .
$$

Combining equations (30) and (33) implies that the secular rate of change of the background angular momentum of the fluid particles may be written as

$$
\begin{aligned}
\frac{d\langle h\rangle}{d t} & \equiv r \sin \theta \hat{\boldsymbol{e}}_{\phi} \cdot \frac{d\langle\boldsymbol{v}\rangle}{d t} \\
& =-r \sin \theta \hat{\boldsymbol{e}}_{\phi} \cdot \frac{(\langle\boldsymbol{v}\rangle \cdot \nabla \boldsymbol{I}+\nabla\langle\boldsymbol{v}\rangle)}{\langle\rho\rangle} \cdot \frac{\partial\left\langle\mathscr{L}_{2}\right\rangle}{\partial\langle\boldsymbol{v}\rangle} .
\end{aligned}
$$

In arriving at equation (34), we take advantage of the $\phi$ independence of all the background fields and of $\left\langle\mathscr{L}_{2}\right\rangle$. There is a subtle point involved in passing from equation (33) to equation (34) which deserves mention. In principle, a second-order secular variation in the background angular momentum might result from the nonvanishing time average of the product of a first-order variation of $r \sin \theta$ and a first order variation of $v$. That this cannot happen here follows from our proof that the lowest order variations in the background state are already of second order.

\footnotetext{
${ }^{1}$ We use $\epsilon^{2} t$ to convey that derivatives with respect to $t$ are of order $\epsilon^{2}$

${ }^{2}$ The separation of fast and slow perturbations is not possible in some small neighborhood of corotation since $\sigma \approx 0$ there.
} 
To evaluate the right-hand side of equation (34), we use

$$
\frac{\partial\left\langle\mathscr{L}_{2}\right\rangle}{\partial\langle\boldsymbol{v}\rangle}=\frac{\partial\left\langle\mathscr{L}_{2}\right\rangle}{\partial\left\langle v_{\phi}\right\rangle} \hat{\boldsymbol{e}}_{\phi}
$$

Thus

$$
\hat{\boldsymbol{e}}_{\phi} \cdot(\langle\boldsymbol{v}\rangle \cdot \nabla) \boldsymbol{I} \frac{\partial\left\langle\mathscr{L}_{2}\right\rangle}{\partial\langle\boldsymbol{v}\rangle}=\frac{\left\langle v_{\phi}\right\rangle}{r \sin \theta} \hat{\boldsymbol{e}}_{\phi} \cdot \frac{\partial}{\partial \phi}\left(\frac{\partial\left\langle\mathscr{L}_{2}\right\rangle}{\partial\left\langle v_{\phi}\right\rangle} \hat{\boldsymbol{e}}_{\phi}\right)=0
$$

since

$$
\hat{\boldsymbol{e}}_{\phi} \cdot \frac{\partial \hat{\boldsymbol{e}}_{\phi}}{\partial \phi}=0, \text { and } \frac{\partial}{\partial \phi}\left(\frac{\partial\left\langle\mathscr{L}_{2}\right\rangle}{\partial\left\langle v_{\phi}\right\rangle}\right)=0
$$

Also

$$
\hat{\boldsymbol{e}}_{\phi} \cdot \nabla\langle\boldsymbol{v}\rangle \cdot \frac{\partial\left\langle\mathscr{L}_{2}\right\rangle}{\partial\langle\boldsymbol{v}\rangle}=\hat{\boldsymbol{e}}_{\phi} \cdot \nabla\langle\boldsymbol{v}\rangle \cdot \frac{\partial\left\langle\mathscr{L}_{2}\right\rangle}{\partial\left\langle v_{\phi}\right\rangle} \hat{\boldsymbol{e}}_{\phi}=0,
$$

since

$$
\hat{\boldsymbol{e}}_{\phi} \cdot \nabla \hat{\boldsymbol{e}}_{\phi} \cdot \hat{\boldsymbol{e}}_{\phi}=0
$$

and

$$
\hat{\boldsymbol{e}}_{\phi} \cdot \nabla\left(\frac{\partial\left\langle\mathscr{L}_{2}\right\rangle}{\partial\left\langle v_{\phi}\right\rangle}\right)=0 .
$$

These steps complete our proof since equation (34) is now seen to read

$$
\frac{d\langle h\rangle}{d t}=0
$$

The astute reader will have noticed that the proof of our theorem does not require knowledge of the form of $\left\langle\mathscr{L}_{2}\right\rangle$ beyond its obvious independence on $t$ and $\phi$. Moreover, unlike the examples discussed by Dewar (1970), it does not depend on any short-wavelength approximation.

Before leaving this section, it is perhaps worth reflecting on the advantages we gain by following Dewar's procedure for splitting the exact state of our fluid system into a background state plus a periodic perturbation. The more conventional approach is to decompose the exact state into the unperturbed state plus an unconstrained perturbation; the secular variation is included as part of the perturbation. To obtain the secular evolution of the system to second-order requires a calculation of the second-order displacement vector, and thus the expansion of the Langrangian density to third order. Furthermore, additional second-order secular variations arise from the nonvanishing time averages of products of first-order pertur- bations. Dewar's method lets one get away with calculating the first-order displacement vector from the second-order part of the Langrangian density.

\section{DISCUSSION}

In taking the time average of the perturbed Lagrangian we are following a procedure similar to that used in celestial mechanics perturbation theory. There, averaging is performed to eliminate short-period terms from the perturbation potential, or disturbing function. Secular perturbations of an orbit are calculated from the partial derivatives of the time-averaged disturbing function with respect to the orbital elements. For a system of small masses in orbit about a large central mass, there is even a theorem analogous to ours. It states that, to second order in the ratio of the masses of small bodies to that of the large central body, the secular variations in semimajor axis vanish. A more detailed comparison reveals additional similarities involving the role of corotation resonances.

Our theorem shows that, away from corotation and in the absence of dissipation, fluid elements preserve their average angular momenta. ${ }^{3}$ Of course, this does not require that the tidal torque vanish. Rather, it implies that internal stresses transport away the angular momentum transferred to the fluid by the external potential. The accumulation of angular momentum can only occur at corotation, or where there is dissipation.

Additional physical insight follows from considering the frequency at which the fluid particles are forced by the tidal perturbation. The inertial frequency of the external potential is $\omega=m \Omega_{p}$, and this is the frequency of the tidal perturbation it forces. However, by virtue of their angular velocities, the fluid particles feel the forces at the Doppler-shifted frequency, $\sigma \equiv$ $m\left(\Omega_{p}-\Omega\right)$. Away from corotation $\sigma \neq 0$, and adiabatic invariance ensures that the wave action, which is proportional to the angular momentum density, is conserved. In quantummechanical language we say that wave quanta cannot be absorbed or emitted except at corotation.

The close relation between conservation of wave action and adiabatic invariance suggests that our theorem, which was proven to second order in the perturbation strength, holds to all orders. A discussion of this point in a related context is given by Dewar (1970).

This research was supported by NSF grant AST 861299 . P. G. is grateful to J. N. Bahcall for his hospitality at the Institute for Advanced Study where this investigation was initiated.

\footnotetext{
${ }^{3}$ We are assuming that the velocity field is laminar.
}

Dewar, R. L. 1970, Phys. Fluids, 13, 2710.

Goldreich, P., and Nicholson, P. 1989, Ap. J., 342, 000

Goldreich, P., and Tremaine, S. 1978, Icarus, 34, 240.

\section{REFERENCES}

Newcomb, W. A. 1962, Nucl. Fusion Suppl., $2,451$.

Whitham, G. B. 1965, J. Fluid Mech., 22, 273.

Zahn, J.-P. 1975. Astr. Ap., 41, 329.

. 1977. Astr. Ap., 57, 383.

Peter Goldreich: Mail Code 170-25, California Institute of Technology, Pasadena, CA 91125

Philip D. Nicholson: Space Sciences Building, Cornell University, Ithaca, NY 14853 